\title{
Aging Affects Bone Marrow Macrophage Polarization: Relevance to Bone Healing
}

\author{
E Gibon $^{1,2,3} \cdot$ F Loi $^{1} \cdot$ Luis A Córdova $^{1,4} \cdot \mathrm{JPajarinen}^{1} \cdot \mathrm{T} \mathrm{Lin}^{1} \cdot \mathrm{L} \mathrm{Lu}^{1} \cdot \mathrm{A} \mathrm{Nabeshima}^{1}$. \\ $\mathrm{Z} \mathrm{Yao}^{1} \cdot$ Stuart B Goodman ${ }^{1,5,6}$
}

Received: 30 March 2016 / Accepted: 6 June 2016 / Published online: 14 June 2016

(C) The Regenerative Engineering Society 2016

\begin{abstract}
Macrophages are an important component of the inflammatory cascade by initiating and modulating the processes leading to tissue regeneration and bone healing. Depending on the local environment, macrophages can be polarized into M1 (pro-inflammatory) or M2 (antiinflammatory) phenotypes. In order to assess the effects of aging on macrophage function, bone marrow macrophage polarization using primary bone marrow macrophages (BMMs) from young (8 weeks old) and aged (72 weeks old) wild-type male $\mathrm{C} 57 \mathrm{BL} / 6 \mathrm{~J}$ mice was analyzed. Fluorescence-activated cell sorting (FACS) analysis (CD11b, iNOS, CD206), qRT-PCR (iNOS, TNF- $\alpha$, CD206, Arginase 1), and ELISA (TNF- $\alpha$, IL-1ra) were performed
\end{abstract}

E Gibon and F Loi contributed equally.

Stuart B Goodman

emmanuel.gibon@gmail.com

1 Department of Orthopaedic Surgery, Stanford University, R116, 300 Pasteur Drive, Stanford, CA 94305, USA

2 Laboratoire de Biomécanique et Biomatériaux Ostéo-Articulaires UMR CNRS 7052, Faculté de Médecine - Université Paris7, 10 avenue de Verdun, 75010 Paris, France

3 Department of Orthopaedic Surgery, Hopital Cochin, APHP, Université Paris5, 27 rue du Faubourg Saint-Jacques, 75014 Paris, France

4 Department of Oral and Maxillofacial Surgery, University of Chile-Conicyt, Santiago, Chile

5 Department of Bioengineering, Stanford University, Stanford, CA, USA

6 Department of Orthopaedic Surgery and (by courtesy) Bioengineering, Stanford University Medical Center Outpatient Center, 450 Broadway St., M/C 6342, Redwood City, CA 94063, USA to compare the M1 and M2 phenotypic markers in young and aged mouse macrophages. Once M1 and M2 macrophage phenotypes were confirmed, the results showed that TNF- $\alpha$ mRNA was significantly upregulated in aged M1s after interferon gamma (INF- $\gamma$ ) exposure. Arginase 1 and CD206 mRNA expression were still upregulated with IL4 stimulation in aged macrophages, but to a lesser extend than those from younger animals. TNF- $\alpha$ secretion was also significantly increased in aged M1s compared to young M1s, following lipopolysaccharide (LPS) exposure. However, the IL-1ra secretion did not increase accordingly in aged mice. The results demonstrate that, compared to younger animals, aging of bone marrow derived macrophages increases the resting levels of oxidative stress, and the ratios of pro- to anti-inflammatory markers. These agerelated changes in macrophage polarization may explain in part the attenuated response to adverse stimuli and delay in processes such as fracture healing seen in the elderly.

\section{Lay Summary}

Bone healing is a complex process that involves both biological and mechanical factors. Macrophages are key cells that regulate the events involved in bone healing, especially the initial inflammatory phase. In this biological cascade of events, macrophages present as different functional phenotypes including uncommitted (M0), pro-inflammatory (M1), and anti-inflammatory (M2), a process called macrophage polarization. A clear understanding of the effects of aging on macrophage polarization is critical to modulating adverse events such as fractures, atraumatic bone loss, and tissue regeneration in an aging population.

Keywords Aging $\cdot$ Bone marrow macrophage $\cdot$ Polarization . Bone healing 


\section{Introduction}

The American population is aging rapidly and people over 65 years of age already represent $13 \%$ of the population [1]. This number is expected to increase as the "Baby Boomer" generation continues to age beyond 65 [2]. The most recent projections have shown that this segment of the population will increase to $25.8 \%$ by 2060 [3]. Complications related to healing of fractures and other inflammatory conditions involving bone represent a large burden for orthopedic surgeons and society. Macrophages have been shown to be key cells in the inflammatory cascade, and are a major component in the events involved in tissue healing and regeneration in diverse tissues and organ systems including bone. Macrophages (M $\Phi)$ initiate the acute inflammatory response which leads to the secretion of proinflammatory factors such as tumor necrosis factor alpha (TNF- $\alpha$ ) and interleukin-1 (IL-1), IL-6, IL-11, and IL-18 [4]. This acute inflammatory response is of paramount importance for bone healing; indeed, the use of nonsteroid anti-inflammatory drugs (NSAIDs) has been associated with an increased incidence of nonunion [5]. Moreover, $\mathrm{M} \Phi$ also secrete a myriad of factors (e.g., fibroblast growth factor (FGF) and platelet-derived growth factor (PDGF)) which are involved in the chemotaxis of mesenchymal stem cells (MSCs) [6]. Inflammation plays a vital role in early fracture healing as shown by Glass et al. [7], who noted that addition of TNF- $\alpha$ at the fracture site accelerated bone healing. Moreover, Gerstenfeld et al. [8] have shown that bone healing was delayed in TNF- $\alpha$-deficient mice $\left(\mathrm{p} 55^{-/} / \mathrm{p} 75^{-/}\right)$. Monocyte chemotactic protein-1 (MCP-1) and monocyte inflammatory protein-1 alpha (MIP-1 $\alpha$ ) are two other essential chemokines secreted by $M \Phi$ that lead to MSC homing and migration to the injury site [9]. $\mathrm{M} \Phi$ are also key cells in other inflammatory orthopedic conditions, for example, particle-induced periprosthetic osteolysis [10].

$\mathrm{M} \Phi$ can be broadly described, as pro-inflammatory $\mathrm{M} 1$, and anti-inflammatory $\mathrm{M} 2$ populations [11]. The local microenvironment drives uncommitted macrophages to either M1 or M2 phenotypes. M1 polarization, also known as classical $\mathrm{M} \Phi$ activation, is induced by such environmental signals as interferon gamma (IFN- $\gamma$ ), toll-like receptor (TLR) signaling [12], and biomaterial wear debris [13]. Essential transcription factors associated with M1 polarization include STAT1 and NF-KB [14]. M2 polarization, also known as alternative $\mathrm{M} \Phi$ activation, is induced by IL4, with STAT6 being the key transcription factor [15]. M1s are characterized by a cytokine release profile of TNF- $\alpha$, IL- 6 , IL-1, IL-12, IL-23, oncostatin M (OSM), and type 1 interferon, with increased expression of iNOS, CCR7, and HLA-DR [16, 17]. It is important to note that iNOS is only expressed in murine M1 macrophages, and similarly Arg1, Fizz1, and Ym1 are murine M2 markers (not human). The M2 cytokine release profile includes IL-4, IL-10, IL-13, and IL-1ra and increased expression of CD206, Ym1, CD163, CCL1, CCL18, FIZZ1, arginase 1 (Arg1), and chitotriosidase [18, 19]. In addition to physiological transition from inflammation to tissue regeneration, this concept of $\mathrm{M} \Phi$ polarization has been shown to be relevant to processes involved in particle-induced periprosthetic osteolysis [20]. Furthermore, uncommitted M0s assume an M2 phenotype more efficiently if first passed through the M1 phenotype [20]. The concept of macrophage polarization is also relevant to fracture healing which includes an initial stage of inflammation prior to later stage of bone formation [4].

$M \Phi$ function and polarization may be impaired with aging. Mahbub et al. [21] polarized splenocytes from young and aged female BABL/c mice. They found decreased IL- $1 \beta$ and TNF- $\alpha$ protein levels in aged M1s, compared to young M1s. Barrett et al. [22] showed an increased response to inflammatory stimuli in aged compared to younger rat M1s, with significantly higher levels of TNF- $\alpha$ RNA. However, no M2 polarization was performed in this study. Thus, the effects of aging on bone marrow $\mathrm{M} \Phi$ polarization have been insufficiently investigated and are still controversial. A clear understanding of the effects of aging on $\mathrm{M} \Phi$ polarization is not only relevant to bone healing; these processes are important to the many different diseases and biological conditions including cancer, atherosclerosis and cardiac disease, neurodegenerative diseases, obesity and insulin resistance, periodontal disease, and others [23]. In the present study, the effects of aging on $\mathrm{M} \Phi$ polarization and subsequent gene expression and protein activity, using primary bone marrowderived $M \Phi$ from young and aged male mice were examined.

\section{Material and Methods}

\section{Mouse Bone Marrow Macrophage (mBMM) Isolation}

Ten C57BL/6J male mice (Jackson Laboratory, Sacramento, CA) were divided into two groups. The experiment was approved by the institution's Administrative Panel for Laboratory Animal Care (APLAC number 9964). All applicable international, national, and/or institutional guidelines for the care and use of animals were followed. Group 1 animals were young mice ( 8 weeks old, $n=5$ ). Group 2 animals were aged mice ( 72 weeks old, $n=5$ ). Mice were euthanized with carbon dioxide gas followed by cervical dislocation. After the mice were sterilized in $70 \%$ ethanol, both of their femora and tibiae were surgically removed. Five milliliters of basal medium (RPMI 1640 medium, $10 \%$ heat-inactivated FBS and $1 \%$ antibiotic/antimycotic (all from Life Technologies, Carlsbad, CA)) was used to flush out the bone marrow with a 25 -gauge needle and syringe. The bone marrow was then filtered through a $70-\mu \mathrm{m}$ strainer, centrifuged $(400 \mathrm{~g}, 10 \mathrm{~min})$, resuspended in ice-cold red blood cell lysis buffer (Sigma-Aldrich, St. Louis, $\mathrm{MO})$, centrifuged again $(400 \mathrm{~g}, 10 \mathrm{~min})$ after addition of basal medium, resuspended in complete M $\Phi$ media (basal medium with $30 \%$ L929 cell-conditioned medium (LCM) and $10 \mathrm{ng} /$ $\mathrm{mL}$ macrophage colony-stimulating factor (MCSF; R\&D 
Systems, Minneapolis, MN)), and plated in T-175 culture flasks (BD, Franklin Lakes, NJ) at a concentration of $5 \times 10^{7}$ cells/ flask. Cells were cultured for 7 days, with a medium change every $2-3$ days to obtain unpolarized $M \Phi$ (referred to hereafter as "M0s").

\section{Macrophage Polarization}

$\mathrm{M} \Phi$ were cultured to $80 \%$ confluency, split, and cultured in 24-well plates at a density of $7.5 \times 10^{4}$ cells per well. $\mathrm{M} \Phi$ were polarized to M0, M1, and M2 phenotypes by exposure to polarization medium for $24 \mathrm{~h}$. M0 polarization medium consisted of complete $M \Phi$ medium without MCSF. To induce $\mathrm{M} 1$ and $\mathrm{M} 2$ polarization, additional $100 \mathrm{ng} / \mathrm{mL}$ lipopolysaccharide (LPS) (Sigma-Aldrich) or $20 \mathrm{ng} / \mathrm{mL} \mathrm{IFN-} \gamma$ (M1) and $20 \mathrm{ng} / \mathrm{mL}$ IL-4 (M2) (all from R\&D Systems) was added to the cultures, respectively. Supernatants were collected after $24 \mathrm{~h}$ and adherent cells were lysed for RNA isolation. The supernatants and cell lysates were stored in $-80^{\circ} \mathrm{C}$ until use.

\section{Characterization of Macrophages}

M0, M1, and M2 M $\Phi\left(7.5 \times 10^{4} /\right.$ well $)$ were cultured in $24-$ well plates in M $\Phi$ medium (RPMI 1640 with $10 \% \mathrm{FBS}, 30 \%$ LCM, $1 \%$ antibiotic/antimycotic) for $24 \mathrm{~h}$ after polarization for real-time quantitative reverse polymerase chain reaction (qRT-PCR) and enzyme-linked immunosorbent assay (ELISA). Cultures in $175-\mathrm{cm}^{2}$ flasks were used for fluorescence-activated cell sorting (FACS). M1 and M2 markers were analyzed with FACS, qRT-PCR, and ELISA.

\section{FACS}

Cells were labeled using the anti-mouse antibodies: CD11bPE (concentration 1:400), iNOS-Alexa Fluor 488 (concentration 1:100), and CD206-APC (concentration 1:100) (Biolegend). We collected 10,000 cell events per sample, and we performed unstained control as well as isotype controls. Labeled cells were examined on the LSR II Analyzer (BD) in the Stanford Shared FACS Facility, and data was analyzed with FlowJo software (Tree Star, Ashland, OR). Dead cells were excluded by ethidium monoazide (EMA) staining, and appropriate isotype controls were used. Quadrants were drawn using the M0 isotope control.

\section{RNA Isolation and qRT-PCR}

Total RNA was extracted using Qiagen's RNeasy Mini Kit (Valencia, CA). The amount of the total RNA was measured with a NanoDrop 1000 spectrophotometer (Thermo Scientific). Total RNA (100 ng/sample) was reverse-transcribed using the High-Capacity cDNA Reverse Transcription Kit from Applied Biosystems (Foster City, CA). qRT-PCR was performed using
TaqMan Gene Expression Master Mix and primer probes for 18 s, iNOS, TNF- $\alpha$, CD206, Arg1, and ABI 7900HT Sequencing Detection System (all from Applied Biosystems). Housekeeping gene 18s was used as the internal control. Relative gene expression level to gene $18 \mathrm{~s}$ was quantified with the comparative $\mathrm{Ct}$ method.

\section{ELISAs}

Supernatants from M0, M1, and M2 cultures were analyzed using commercially available ELISA kits for TNF- $\alpha$ and IL1ra (all from R\&D Systems).

\section{Statistical Analysis}

Statistical analyses were performed with GraphPad Prism version 6.04 for Windows (GraphPad Software, San Diego, CA). Group analyses were conducted with two-way ANOVA considering two variables (age and polarization stimuli). $p<0.05$ was considered statistically significant.

\section{Results}

\section{Aged M0s Overexpress Both M1 and M2 Surface Markers}

As seen in Fig. 1, quantification of M $\Phi$ phenotype by FACS showed that aged untreated M $\Phi$ (M0s) are $\mathrm{iNOS}^{\text {high/ }}$ CD206 ${ }^{\text {high }}$ compared with young untreated M $\Phi(\mathrm{M} 0 \mathrm{~s})$, which are $\mathrm{iNOS}^{\text {low }} / \mathrm{CD} 206^{\text {low }}$ (68/46.30 vs. 27.63/19.43\%). Moreover, after polarization, aged M1s had a higher expression of CD206 both after LPS (35.24 vs. $20.22 \%$ ) and IFN- $\gamma$ ( 37.17 vs. $14.73 \%$ ) polarization compared to young M1s, whereas iNOS expression was similar between the young and aged cells. There was no difference between young and aged M2 polarized cells.

\section{Aged M1s Upregulate TNF- $\alpha$; Aged M2s Downregulate Arg1 and CD206}

After polarization with IFN- $\gamma$, aged M1s responded by upregulating TNF- $\alpha$ mRNA expression compared to young M1s. Polarization with LPS also increased TNF- $\alpha$ mRNA expression in aged M1s, but this did not reach statistical significance when compared with young M1s. iNOS mRNA expression was also upregulated with IFN stimulus in aged M1s, but interestingly, this value was lower than in young M1s (Fig. 2). There was no significant difference for iNOS and TNF- $\alpha$ mRNA expression after polarization with LPS between young and aged M1s.

After polarization with IL-4, young M2s significantly increased Arg1 or CD206 mRNA expression compared to aged M2s (Fig. 2). 
A

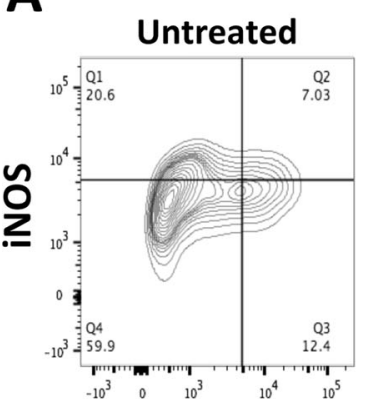

CD206

B

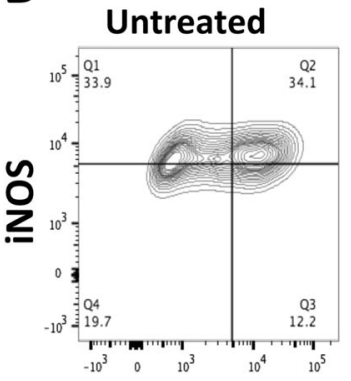

CD206

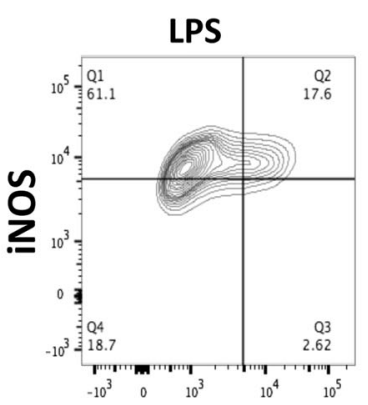

CD206

LPS

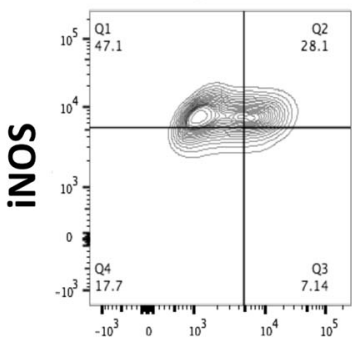

CD206

Fig. 1 Characterization of mouse bone marrow-derived macrophages (a young; $\mathbf{b}$ aged) after polarization. Most of the aged M0s are both iNOS+ and CD206 + where as most of the young M0s are iNOS- and CD206-.

IFN- $\gamma$

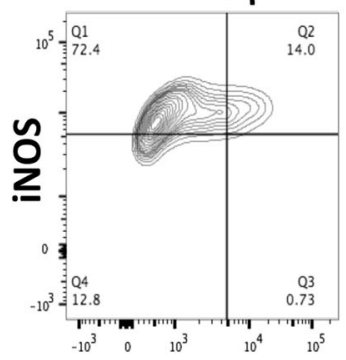

CD206

IFN- $\gamma$

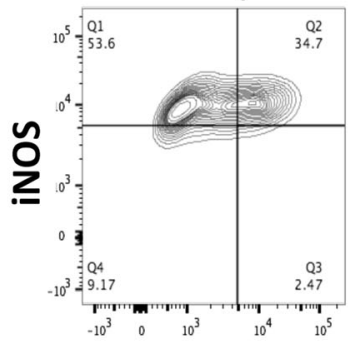

CD206

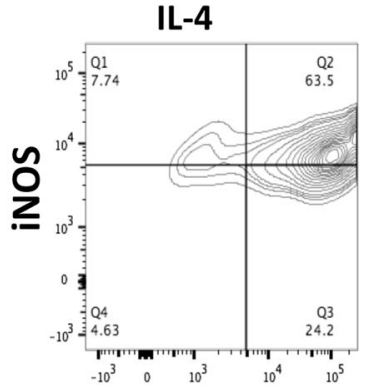

CD206

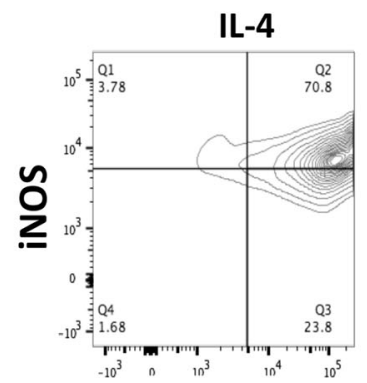

CD206

There was no difference between young and aged M2 polarized cells and no difference between LPS and IFN- $\gamma$ induced M1 polarization

Fig. 2 qRT-PCR for iNOS, TNF$\alpha$, and arginase 1 , and CD206 after polarization. Aged M1s significantly overreacted to polarization stimuli with IFN- $\gamma$ with increased TNF- $\alpha$ mRNA expression underlying a higher sensitivity to the microenvironment. CD206 and arginase 1 mRNA are downregulated in aged M2s after IL-4 polarization compared to young M2s. Data reported as mean \pm standard error of the mean. Fold refers to change over M0
iNOS
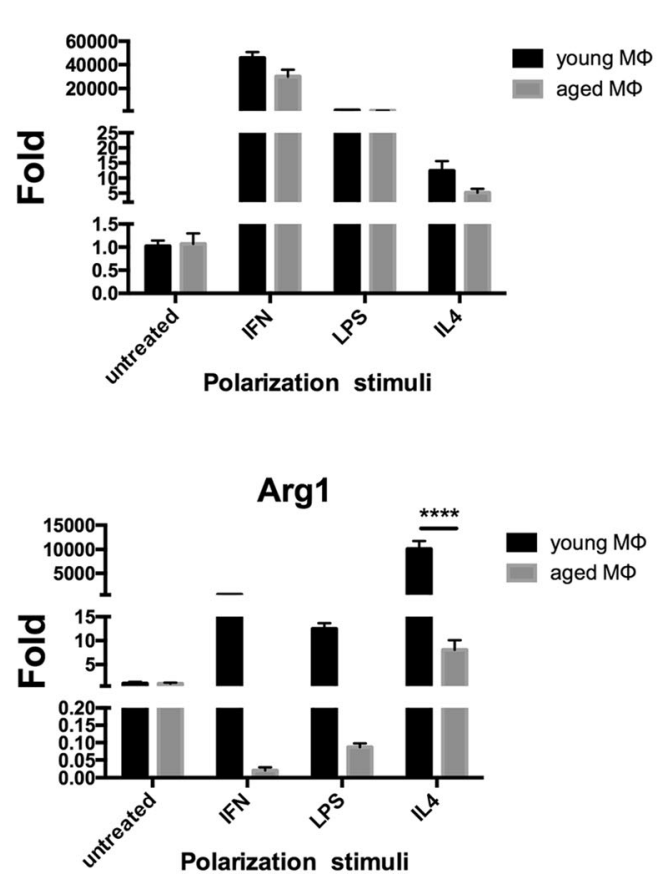

TNF- $\alpha$

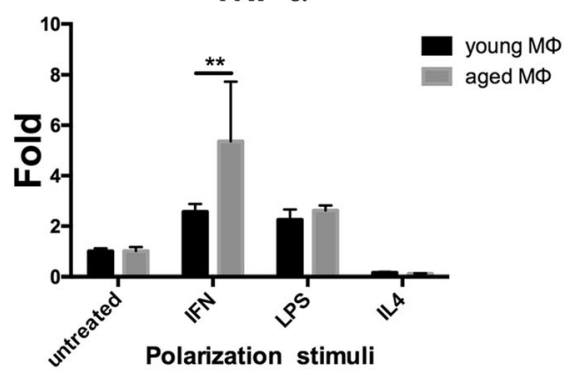

CD206

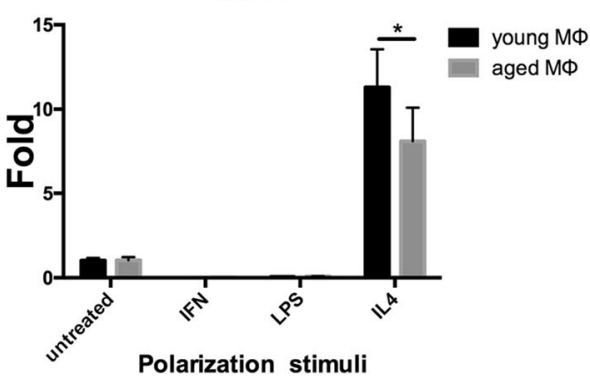


Aged M1s Increase Secretion of TNF- $\alpha$ Protein with no Negative Feedback

As seen in Fig. 3, secreted TNF- $\alpha$ protein was significantly increased in aged M1s compared to young M1s phenotypes after polarization with LPS but not with IFN- $\gamma$. IL-1ra was significantly lower in aged M1s polarized with LPS compared to young M1s polarized with LPS indicating an inability to induce negative feedback (Fig. 3). There was a significant difference between young and aged M2s regarding IL-1 ra secretion $(245.60 \pm 12.07$ vs. $199.50 \pm 5.39 \mathrm{pg} / \mathrm{ml}$ respectively, $p<0.05)$.

\section{Discussion}

The aim of the current study was to determine whether aging impairs polarization of bone marrow-derived macrophages, cells important to inflammation, tissue regeneration, and bone healing. Fractures are among the most common orthopedic problems that require medical intervention, particularly in the elderly. Inflammation is the first stage in the healing of fractures and bone injuries. However, most studies regarding the biology of fracture healing have been performed using young mice (8-10 weeks old). This applies to studies investigating other inflammatory conditions, such as infection, osteonecrosis, periprosthetic osteolysis, and others. Therefore, to better understand the bone biology in the elderly, in vivo models with aged animals would be more appropriate. Consistent with this idea, the decision was made to compare young male C57BL/6J (8-10 weeks old) mice to aged male C57BL/6J mice (70-72 weeks old). In addition to age, gender is an important variable [24] and needs to be further studied.

In this study, aged $\mathrm{M} \Phi$ responded somewhat differently to polarizing stimuli compared to young cells. The change in responsiveness to inflammatory stimuli in aged $M \Phi$ is often referred to as "inflamm-aging" [25]. Previous studies found elevated pro-inflammatory factor levels with age [26]. High levels of TNF- $\alpha$, iNOS, IL- $1 \beta$, and IFN- $\gamma$ have been observed when aged M $\Phi$ were challenged with IFN- $\gamma$ or LPS $[22,27,28]$. These findings suggest that aged $M \Phi$ remain in a preactivated resting state that enhances their responsiveness to exposure of pro-inflammatory stimuli [29]. Loi et al. [30] have shown that modulation of macrophage phenotype from M1 to M2 enhances osteogenic ability of MC3T3 cells in an in vitro coculture model. The present study showed that the majority of the aged M0s are both iNOS+ and CD206+. Not only is the expression of both iNOS and CD206 increased in aged M0s compared to young M0s, but most notably, the number of M0s that are doubly positive for both iNOS and CD206 is increased in aged M0s. This result underscores a heightened preactivated resting state in aged compared to younger resting

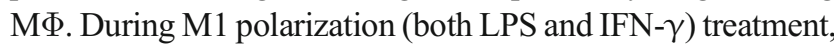
an increase in the overall amount of cells expressing iNOS was observed. Interestingly this LPS or IFN- $\gamma$ induced increase in iNOS expression was mainly due to an increase in the number of single iNOS-positive cells, while the percentage of cells double positive for iNOS and CD206 remained almost unchanged. This phenomenon was observed both in young and aged macrophages and suggests that LPS or IFN- $\gamma$ treatment does not lead to upregulation of CD206. Indeed corresponding results were observed in the qRT-PCR where neither LPS nor IFN- $\gamma$ upregulated CD206 expression. In contrast, M2 polarization with IL-4 treatment led to strong upregulation of CD206 as expected but surprisingly, also iNOS expression was upregulated and most of these cells were also iNOS positive. It is unclear why IL-4 treatment also increases iNOS expression but this seems to be supported also by the qRT-PCR data. Furthermore, the present study showed that M1 polarization is impaired with aging; despite the fact that aged $M \Phi$ expressed the iNOS surface marker similar to young M1s, aged M1s also expressed CD206 ${ }^{\text {high }}$. Moreover, IFN and LPS stimulation of M0s did not lead to identical macrophage phenotypic M1 profiles: aged M1s stimulated with LPS produced significantly more TNF- $\alpha$ than aged M1s stimulated with IFN- $\gamma$.

The present results showed that when aged M $\Phi$ were challenged with IFN- $\gamma$, aged M1s had significantly higher levels of TNF- $\alpha$ mRNA expression compared to young M $\Phi$. This is consistent with the hypothesis that aged $M \Phi$ remain in a preactivated "resting" state. However, the TNF- $\alpha$ secretion was greatly increased after LPS stimulation in aged $M \Phi$, suggesting that a strong polarization stimulus mimicking infection enhances the responsiveness of aged M $\Phi$. For M2s, the
Fig. 3 TNF- $\alpha$ secretion is significantly increased in aged M1s polarized with LPS whereas the IL-1 ra increase was significantly lower in aged M1s polarized with LPS. Aged M1s have a dysregulated negative feedback control over inflammation. Data reported as mean \pm standard error of the mean
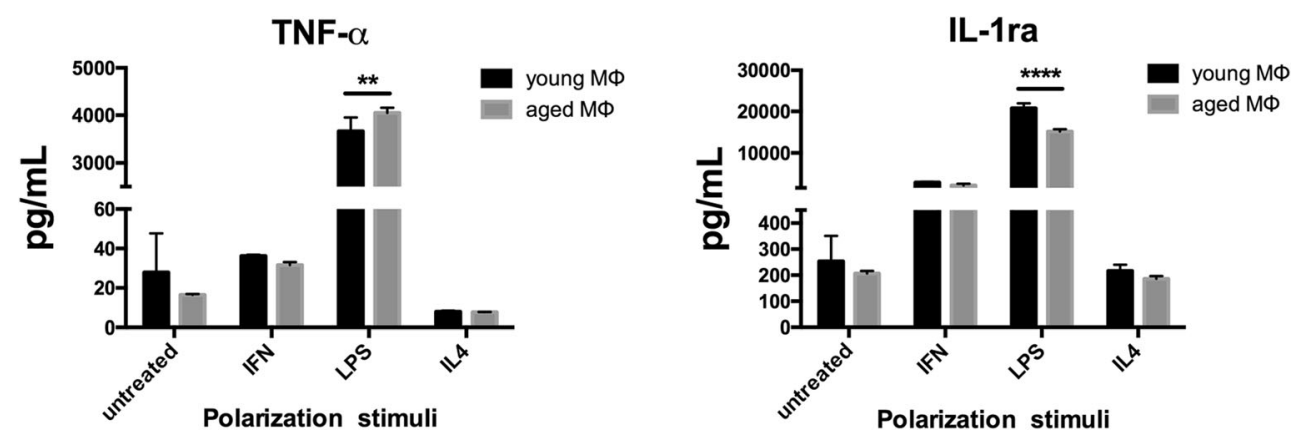
results show that $\operatorname{Arg} 1$ mRNA regulation is greatly impacted by macrophage aging, while CD206 mRNA is impacted less. This may be due to signaling pathway abnormalities in IL-4 receptor binding, whereby Arg1 production is blocked in aged $\mathrm{M} \Phi$ while the pathway leading to CD206 production is still functional. This failure to secrete $\operatorname{Arg} 1$ in aged $M \Phi$ is problematic as Salimudin at al. showed that Arg1 functions to downregulate endotoxin-induced nitric oxide production, thus allowing the healing process to commence [31]. Moreover, the ability of aged $\mathrm{M} \Phi$ to subsequently secrete inflammatory proteins was increased. Although TNF- $\alpha$ secretion was significantly increased in aged M1s after LPS stimulation, the IL1ra secretion was not significantly increased accordingly in these cells, suggesting a dysregulation in aged M1s with an inability to modulate increased TNF- $\alpha$ release following LPS exposure. These results were unexpected as IL-1ra is an antiinflammatory cytokine and is usually higher in M2s [32, 33]. This decreased negative feedback control over inflammation in aged M1s may explain the high inflammatory state commonly found in the elderly [34-36].

This study has some limitations. First, the analysis examined a limited number of M1 and M2 phenotypic markers at one time point; considering the complexity of macrophage biology, one should consider this study preliminary. Second, this study involved the use of murine cells to simulate biological processes occurring in humans. Furthermore, only macrophages were examined. These cells interact with other cells lineages in vivo, such as mesenchymal stem cells (MSCs), lymphocytes, fibroblasts, and many other cell types. In consideration of this fact, further studies are necessary to investigate the effect of aging on cocultured aged and young $M \Phi$ and cells such as MSCs, to further understand the effects of inflammatory cells on bone formation. Limited studies have been reported on this subject [37-40]. Third, although this study was very labor intensive, the experiment was performed only twice and the cells were pooled together. Gender effects may play an important role as well and need to be further studied.

\section{Conclusion}

This study demonstrates that bone marrow-derived macrophage polarization in mice is altered with aging. A large number of double iNOS/CD206 positivity was found in aged M0s and a lack of Arg1/CD206 mRNA expression in aged M2s. Aged M1s significantly increased TNF- $\alpha$ mRNA expression and protein secretion following IFN- $\gamma$ and LPS exposure, respectively. The usual negative feedback system appears to be dysregulated with aging as aged M1s could not increase the IL-1ra secretion after LPS exposure. These differences could become more pronounced with advances age. $M \Phi$, key cells in both bone healing and inflammatory processes demonstrate impaired polarization and cytokine secretion with aging. This fact presents an opportunity for modulation of the biological microenvironment to optimize bone healing and repair in the elderly.

Acknowledgments The authors would like to thank the Ellenburg Chair in Surgery, the French Society of Orthopedic Surgery and Traumatology (SOFCOT), and the Philippe Foundation Inc.

\section{Compliance with Ethical Standards}

Conflict of Interest The authors declare that they have no conflict of interest.

Research Involving Human Participants and/or Animals All applicable international, national, and/or institutional guidelines for the care and use of animals were followed. This article does not contain any studies with human participants performed by any of the authors.

Funding This research was supported by grants 2R01AR055650 and 1R01AR063717 from the National Institute of Arthritis and Musculoskeletal and Skin Diseases (NIAMS) at the National Institutes of Health (NIH).

\section{References}

1. UScensus. U.S. Department of Commerce, Economics and Statistics Administration, U.S. Census Bureau, Whashington. 2010. http://www.census.gov/prod/cen2010/briefs/c2010br-09. pdf. Accessed 29 July 2015.

2. Iorio R, Robb WJ, Healy WL, Berry DJ, Hozack WJ, Kyle RF, et al. Orthopaedic surgeon workforce and volume assessment for total hip and knee replacement in the United States: preparing for an epidemic. J Bone Joint Surg Am. 2008;90(7):1598-605.

3. UScensus. U.S. Department of Commerce, Economics and Statistics Administration, U.S. Census Bureau, Whashington. 2015. http://www.census.gov/content/dam/Census/library/publications/2015 /demo/p25-1143.pdf. Accessed 29 July 2015.

4. Marsell R, Einhorn, TA. The biology of fracture healing. Injury. 2011.

5. Giannoudis PV, MacDonald DA, Matthews SJ, Smith RM, Furlong AJ, De Boer P. Nonunion of the femoral diaphysis. The influence of reaming and non-steroidal anti-inflammatory drugs. J Bone Joint Surg (Br). 2000;82(5):655-8.

6. Barnes GL, Kostenuik PJ, Gerstenfeld LC, Einhorn TA. Growth factor regulation of fracture repair. J Bone Miner Res. 1999;14(11):1805-15.

7. Glass GE, Chan JK, Freidin A, Feldmann M, Horwood NJ, Nanchahal J. TNF-alpha promotes fracture repair by augmenting the recruitment and differentiation of muscle-derived stromal cells. Proc Natl Acad Sci U S A. 2011;108(4):1585-90.

8. Gerstenfeld LC, Cho TJ, Kon T, Aizawa T, Cruceta J, Graves BD, et al. Impaired intramembranous bone formation during bone repair in the absence of tumor necrosis factor-alpha signaling. Cells Tissues Organs. 2001;169(3):285-94.

9. Ito H. Chemokines in mesenchymal stem cell therapy for bone repair: a novel concept of recruiting mesenchymal stem cells and the possible cell sources. Mod Rheumatol. 2011;21(2):113-21. 
10. Nich C, Goodman SB. Role of macrophages in the biological reaction to wear debris from joint replacements. J Long-Term Eff Med Implants. 2014;24(4):259-65.

11. Davies LC, Jenkins SJ, Allen JE, Taylor PR. Tissue-resident macrophages. Nat Immunol. 2013;14(10):986-95.

12. Valladares RD, Nich C, Zwingenberger S, Li C, Swank KR, Gibon E, et al. Toll-like receptors- 2 and 4 are overexpressed in an experimental model of particle-induced osteolysis. J Biomed Mater Res A. 2014;102(9):3004-11.

13. Tuan RS, Lee FY, Konttinen YT, Wilkinson JM, Smith RL. What are the local and systemic biologic reactions and mediators to wear debris, and what host factors determine or modulate the biologic response to wear particles? J Am Acad Orthop Surg. 2008;16(Suppl 1):S42-8.

14. Lin T-h, Tamaki Y, Pajarinen J, Waters HA, Woo DK, Yao Z, et al. Chronic inflammation in biomaterial-induced periprosthetic osteolysis: NF-kB as a therapeutic target. Acta Biomater. 2014;10(1):1-10.

15. Murray PJ, Allen JE, Biswas SK, Fisher EA, Gilroy DW, Goerdt S, et al. Macrophage activation and polarization: nomenclature and experimental guidelines. Immunity. 2014;41(1):14-20.

16. Martinez FO, Sica A, Mantovani A, Locati M. Macrophage activation and polarization. Front Biosci. 2008;13:453-61.

17. Guihard P, Boutet M-A. Brounais-Le Royer Bnd, Gamblin A-L, Amiaud Jrm, Renaud a et al. Oncostatin $\mathrm{m}$, an inflammatory cytokine produced by macrophages, supports intramembranous bone healing in a mouse model of tibia injury. Am J Pathol. 2015;185(3):765-75.

18. Maresz K, Ponomarev ED, Barteneva N, Tan Y, Mann MK, Dittel $\mathrm{BN}$. IL-13 induces the expression of the alternative activation marker Ym1 in a subset of testicular macrophages. J Reprod Immunol. 2008;78(2):140-8.

19. Nair MG, Du Y, Perrigoue JG, Zaph C, Taylor JJ, Goldschmidt M, et al. Alternatively activated macrophage-derived RELM-\{alpha\} is a negative regulator of type 2 inflammation in the lung. J Exp Med. 2009;206(4):937-52.

20. Rao AJ, Gibon E, Ma T, Yao Z, Smith RL, Goodman SB. Revision joint replacement, wear particles, and macrophage polarization. Acta Biomater. 2012;8(7):2815-23.

21. Mahbub S, Deburghgraeve CR, Kovacs EJ. Advanced age impairs macrophage polarization. J Interf Cytokine Res. 2012;32(1):18-26.

22. Barrett JP, Costello DA, O'Sullivan J, Cowley TR, Lynch MA. Bone marrow-derived macrophages from aged rats are more responsive to inflammatory stimuli. J Neuroinflammation 2015;12.

23. Brown BN, Ratner BD, Goodman SB, Amar S, Badylak SF. Macrophage polarization: an opportunity for improved outcomes in biomaterials and regenerative medicine. Biomaterials. 2012;33(15):3792-802.

24. Nich C, Rao AJ, Valladares RD, Li C, Christman JE, Antonios JK, et al. Role of direct estrogen receptor signaling in wear particleinduced osteolysis. Biomaterials. 2013;34(3):641-50.

25. Baylis D, Bartlett DB, Patel HP, Roberts HC. Understanding how we age: insights into inflammaging. Longev Healthspan. 2013;2(1):8.
26. Stranks AJ, Hansen AL, Panse I, Mortensen, M, Ferguson, DJP, Puleston, DJ et al. Autophagy controls acquisition of aging features in macrophages. J Innate Immun. 2015.

27. Wang Y, Wehling-Henricks M, Samengo G, Tidball JG. Increases of M2a macrophages and fibrosis in aging muscle are influenced by bone marrow aging and negatively regulated by muscle-derived nitric oxide. Aging Cell. 2015.

28. Cecilio CA, Costa EH, Simioni PU, Gabriel DL, Tamashiro WMSC. Aging alters the production of iNOS, arginase and cytokines in murine macrophages. Braz J Med Biol Res. 2011;44(7):671-81.

29. Smallwood HS, Lopez-Ferrer D, Squier TC. Aging enhances the production of reactive oxygen species and bactericidal activity in peritoneal macrophages by upregulating classical activation pathways. Biochemistry. 2011;50(45):9911-22.

30. Loi F, Córdova LA, Zhang R, Pajarinen J, Lin T-H, Goodman SB, et al. The effects of immunomodulation by macrophage subsets on osteogenesis in vitro. Stem Cell Res Ther. 2016;7:15.

31. Salimuddin N, Nagasaki A, Gotoh T, Isobe H, Mori M. Regulation of the genes for arginase isoforms and related enzymes in mouse macrophages by lipopolysaccharide. Am J Phys. 1999;277(1 Pt 1): E110-7.

32. Mantovani A, Biswas SK, Galdiero MR, Sica A, Locati M Macrophage plasticity and polarization in tissue repair and remodelling. J Pathol. 2013;229(2):176-85.

33. Mantovani A, Sica A, Sozzani S, Allavena P, Vecchi A, Locati M. The chemokine system in diverse forms of macrophage activation and polarization. Trends Immunol. 2004;25(12):677-86.

34. Wåhlin-Larsson B, Carnac G, Kadi F. The influence of systemic inflammation on skeletal muscle in physically active elderly women. Age (Dordr). 2014;36(5):9718.

35. Hubbard RE, Woodhouse KW. Frailty, inflammation and the elderly. Biogerontology. 2010;11(5):635-41.

36. De Martinis M, Franceschi C, Monti D, Ginaldi L. Inflammation markers predicting frailty and mortality in the elderly. Exp Mol Pathol. 2006;80(3):219-27.

37. Zachos C, Steubesand N, Seekamp A, Fuchs S, Lippross S. Cocultures of programmable cells of monocytic origin and mesenchymal stem cells do increase osteogenic differentiation. J Orthop Res. 2014;32(10):1264-70.

38. Cho D-I, Kim MR, H-y J, Jeong HC, Jeong MH, Yoon SH, et al. Mesenchymal stem cells reciprocally regulate the M1/M2 balance in mouse bone marrow-derived macrophages. Exp Mol Med. 2014;46.

39. Maggini J, Mirkin G, Bognanni I, Holmberg J, Piazzon IM, Nepomnaschy I et al. Mouse bone marrow-derived mesenchymal stromal cells turn activated macrophages into a regulatory-like profile. PLoS ONE. 2010;5(2).

40. Vi L, Baht GS, Whetstone H, Ng A, Wei Q, Poon R, et al. Macrophages promote osteoblastic differentiation in-vivo: implications in fracture repair and bone homeostasis. J Bone Miner Res. 2015;30(6):1090-102. 